\title{
The Chlorine Reference Value in Whole Blood using ANAA
}

\author{
Laura Cristina Oliveira, Cibele Bugno Zamboni, \\ Instituto de Pesquisas Energéticas e Nucleares, Caixa Postal 11049, 05422-970, São Paulo, SP, Brazil \\ Patricia da Silva Lins, and Maria Regina Andrade de Azevedo e Oliveira \\ Universidade de Santo Amaro - UNISA, São Paulo, SP, Brazil
}

Received on 1 August, 2005

\begin{abstract}
The Absolute Neutron Activation Analysis (ANAA) technique was used to estimate the reference value for $\mathrm{Cl}$ in whole blood of healthy group, selected from blood banks at São Paulo city, to provide information which can help in diagnosis of patients. The results show that the indicative interval obtained for $\mathrm{Cl}$ in serum from the nuclear analysis $\left(3.41-3.69 \mu g \cdot \mu l^{-1}\right)$ is in agreement with the reference value adopted $\left(3.44-3.76 \mu g \cdot \mu l^{-1}\right)$ in addition, an indicative interval for $\mathrm{Cl}\left(2.75-2.95 \mu \mathrm{g} \cdot \mu l^{-1}\right)$ could be proposed for whole blood. The advantages and limitations of using this nuclear methodology were discussed.
\end{abstract}

\section{INTRODUCTION}

The reference values for trace elements in human clinical specimens can facilitate the interpretation of data deriving from clinical practice because they reflect the findings in a select group of individuals [1]. Several clinical hematological examinations, some of them involving the elements' concentration such as $\mathrm{Cl}$ (Cloremia), $\mathrm{K}$ (Calemia), $\mathrm{Na}$ (Natremia), $\mathrm{Fe}$ (Serum Iron, Ferritin, and TIBC - Total Iron Binding Capacity), for example, are very important to identify anomalies in human being body organs. But, to perform these conventional analysis the procedure needs the separation of serum-plasma, which demands time and it is expensive because different apparatus must be used $[2,3]$. In this work, our purpose is to investigate the performance of the nuclear methodology, specifically the Absolute Neutron Activation Analysis (ANAA), to perform hematological examinations in human being using whole blood.

This study is part of a larger project, Determination of reference values for concentrations of trace elements in human blood using nuclear methodology, at the present in development at IPEN in collaboration with Blood Banks and Hematological Laboratories from different regions of Brazil. Firstly, this nuclear methodology was applied with success to investigated elements concentration in whole blood, urine and also in several body organs of small and medium-sized animals resulting in an agile and economic way to perform clinical investigations [4-10]. Now, we intend to use this nuclear tool to perform hematological analyses in human beings.

The necessity to obtain the reference value is related to the fact that the conventional clinical analyses are performed using serum or plasma, consequently there are no reference value established for whole blood. To perform these measurements a group (male and female blood donators), age between 25 and 60 years at 50 and $85 \mathrm{~kg}$, were selected from blood banks. In this study we choose the element $\mathrm{Cl}$ to be first analyzed because it takes part in the human being metabolism and its important for preservation of the osmotic and acid - base equilibrium of human fluids, for that reason its variations are generally related to several pathological processes. Besides, their nuclear properties such as thermal cross section, half live and isotopic fraction are very favorable to perform neutron activation.

For the development of this investigation the Chlorine concentration in serum was first analyzed, by using ANAA, for comparing the nuclear results with the reference value adopted [2] and for checking the accuracy and precision of this methodology. After that, this methodology was applied to analyze $\mathrm{Cl}$ in whole blood for the proposition of an indicative interval for its reference value.

\section{EXPERIMENTAL PROCEDURE}

In this study the samples came from Blood Bank Paulista at São Paulo city. About $2 \mathrm{ml}$ of whole blood was collected in a vacuum plastic tubing $(15 \mathrm{~mm}$ long and diameter at about $1.0 \mathrm{~mm}$ ) attached to the donator's arm. Immediately after the collection, before its coagulation, a small quantity (50 to $100 \mu \mathrm{l})$ was transferred to the filter paper using a calibrated micropipette and dried for few minutes using an infrared lamp. The biological material yet in the plastic tubing was then centrifuged for the serum sample preparation following the same procedure. It is important to emphasize that the amount of biological material draw up in the filter paper is less than $2 \mathrm{~cm}^{2}$, and that the samples can be storage without any refrigeration.

To determine the concentration of the elements in whole blood or in serum each biological sample was sealed into individual polyethylene bag, together with the Au detector (small metallic foil $\sim 1 \mathrm{mg}$ ) used for measurement of the flux distribution [11], and irradiated for 5 minutes in a pneumatic station in the nuclear reactor (IEA-R1, 2MW, pool type) at IPEN, allowing the simultaneous activation of these materials. Using this procedure the $\gamma$-ray activity induced in the Au detector as well as in the biological sample were obtained under the exact same irradiation conditions. After the irradiation procedure, the activated materials (blood or serum and $\mathrm{Au}$ ) were gammacounted using a HPGe Spectrometer of High Energy Resolution and the areas of the peaks, corresponding to gamma transitions related to the nuclides of interest, were evaluated. The gamma spectra analysis evaluation was performed using 


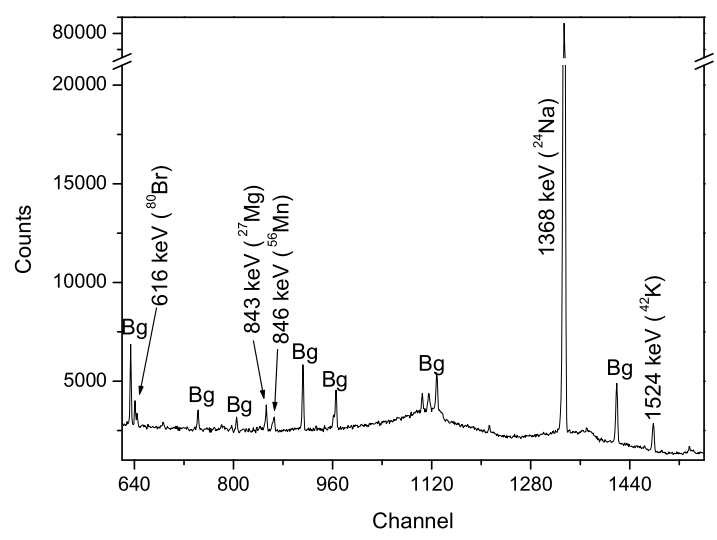

FIG. 1: Partial $\gamma$-ray spectrum of whole blood sample, taken at 5 minutes of irradiation time. Bg indicates peaks occurring in natural background.

the IDEFIX computer software [12] and the calculation of the concentration for each element was obtained from software developed by Medeiros [13]. In this investigation a total of 26 samples for serum and whole blood, respectively, were collected in replicate. The irradiation time of 5 minutes, counting time of 1 minutes for the Gold activation detector and 10 minutes for the biological sample and background radiation $(\mathrm{Bg})$ allowed us to conclude the analysis of each sample (whole blood or serum) in about half hour.

\section{RESULTS}

The $\mathrm{Cl}$ concentration in serum and whole blood are shown in Table I and the gamma ray spectrum of whole blood in Figs. 1 and 2. All the results were obtained by analyzing replicate samples and the indicative interval for the reference values by using the median value considering one SD. Although the concentration of D3, D8, D13 and D14 samples have been determined these results were not considered in our calculation because, according to the selection performed by the blood bank, these donators were not completely healthy. In the figure 3 the Chlorine's concentration results in serum are shown together with the reference values [2], included for comparison. In this figure we can also notice that the concentration's results of the samples not selected (D3, D8, D13 and D14) do not belong to the reference value showing the sensibility of the method. In figure 4 the Chlorine's concentration results in whole blood are shown where the indicative interval, obtained in this study, was included.

According to figures 1 and 2 , besides $\mathrm{Cl}\left(\mathrm{T}_{1 / 2} \sim 37 \mathrm{~min}\right)$, some other elements are also activated in whole blood, such as ${ }^{28} \mathrm{Al}\left(\mathrm{T}_{1 / 2} \sim 3 \mathrm{~min}\right),{ }^{80} \mathrm{Br}\left(\mathrm{T}_{1 / 2} \sim 18 \mathrm{~min}\right),{ }^{49} \mathrm{Ca}\left(\mathrm{T}_{1 / 2} \sim 9 \mathrm{~min}\right)$, ${ }^{42} \mathrm{~K}\left(\mathrm{~T}_{1 / 2} \sim 12 \mathrm{~h}\right),{ }^{27} \mathrm{Mg}\left(\mathrm{T}_{1 / 2} \sim 9.5 \mathrm{~min}\right),{ }^{56} \mathrm{Mn}\left(\mathrm{T}_{1 / 2} \sim 2.5 \mathrm{~h}\right)$ and ${ }^{24} \mathrm{Na}\left(\mathrm{T}_{1 / 2} \sim 15 \mathrm{~h}\right)$, suggesting that these elements' concentrations can also be measured using the same irradiation

\begin{tabular}{c|c|c|c} 
Sample Code & $\begin{array}{c}\text { Neutron Flux } \\
\left(\times 10^{11} n \cdot \mathrm{cm}^{-2} \cdot s^{-1}\right)\end{array}$ & $\begin{array}{c}\mathrm{Cl}\left(\mu g \cdot \mu l^{-1}\right) \\
\text { serum } \\
3.44-3.76\end{array}$ & $\begin{array}{c}\mathrm{Cl}\left(\mu g \cdot \mu l^{-1}\right) \\
\text { whole blood } \\
b_{3.41-3.69}\end{array}$ \\
\hline & & ${ }^{b} 2.75-2.95$ \\
\hline D1 & 1.16 & $3.36 \pm 0.11$ & $2.87 \pm 0.18$ \\
D2 & 6.51 & $3.52 \pm 0.13$ & $2.82 \pm 0.17$ \\
${ }^{n s}$ D3 & 1.27 & $2.99 \pm 0.14$ & $3.25 \pm 0.20$ \\
D4 & 6.08 & $3.77 \pm 0.11$ & $2.74 \pm 0.17$ \\
D5 & 4.85 & $3.42 \pm 0.12$ & $3.04 \pm 0.18$ \\
D6 & 4.99 & $3.96 \pm 0.15$ & $2.9 \pm 0.18$ \\
D7 & 1.38 & $3.37 \pm 0.14$ & $2.94 \pm 0.18$ \\
${ }^{n s}$ D8 & 1.49 & $2.99 \pm 0.18$ & $2.32 \pm 0.14$ \\
D9 & 1.35 & $3.26 \pm 0.19$ & $2.73 \pm 0.17$ \\
D10 & 4.9 & $3.32 \pm 0.20$ & $2.80 \pm 0.16$ \\
D11 & 3.34 & $3.76 \pm 0.13$ & $2.91 \pm 0.1$ \\
D12 & 3.34 & $3.77 \pm 0.11$ & $2.92 \pm 0.11$ \\
${ }^{n s}$ D13 & 1.17 & $2.89 \pm 0.14$ & $2.74 \pm 0.1$ \\
${ }^{n s}$ D14 & 1.21 & $2.99 \pm 0.17$ & $3.10 \pm 0.11$ \\
D15 & 1.18 & $3.44 \pm 0.17$ & $2.82 \pm 0.10$ \\
D16 & 5.99 & $3.76 \pm 0.11$ & $3.01 \pm 0.11$ \\
D17 & 6.11 & $3.65 \pm 0.13$ & $2.77 \pm 0.11$ \\
D18 & 4.8 & $3.32 \pm 0.11$ & $2.84 \pm 0.10$ \\
D19 & 3.15 & $3.44 \pm 0.12$ & $2.62 \pm 0.09$ \\
D20 & 4.23 & $3.76 \pm 0.14$ & $2.91 \pm 0.10$ \\
D21 & 4.96 & $3.45 \pm 0.12$ & $2.74 \pm 0.10$ \\
D22 & 3.83 & $3.71 \pm 0.13$ & $2.88 \pm 0.10$ \\
D23 & 4.29 & $3.53 \pm 0.13$ & $2.83 \pm 0.11$ \\
D24 & 4.24 & $3.49 \pm 0.13$ & $3.11 \pm 0.11$ \\
D25 & 6.02 & $3.52 \pm 0.13$ & $2.92 \pm 0.11$ \\
D26 & 3.56 & $3.61 \pm 0.13$ & $2.86 \pm 0.10$ \\
& & &
\end{tabular}

TABLE I: The $\mathrm{Cl}$ concentration in serum and whole blood by using the ANAA.

* associated uncertainly $<4 \%$;

${ }^{a}$ reference value [2];

${ }^{b}$ indicative interval proposed in this work;

${ }^{n s}$ samples not selected.

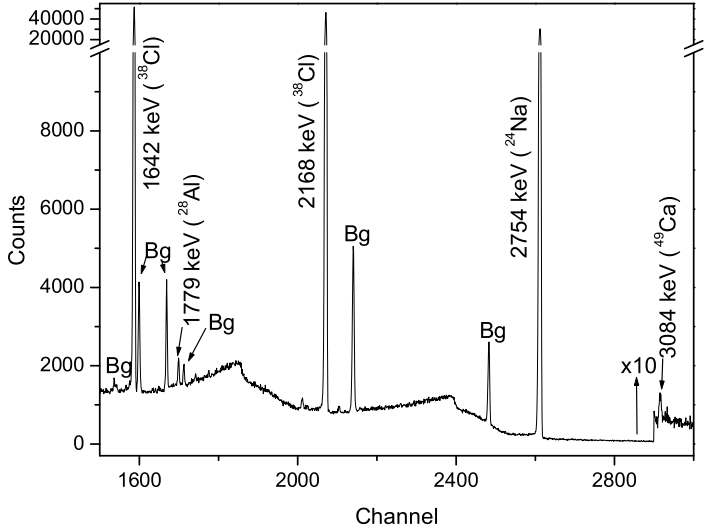

FIG. 2: Partial $\gamma$-ray spectrum of whole blood sample, taken at $5 \mathrm{mi}-$ nutes time irradiation. $\mathrm{Bg}$ indicates peaks occurring in natural background.

condition. We performed preliminary calculations but the elements $\mathrm{Ca}, \mathrm{Mg}$ and $\mathrm{Mn}$, although have been activated, have poor counting statistics so we decide to increase the counting time to 20 minutes and, although few samples have been analyzed, the results showing that all elements activated can also be determined with enough statistic using the same irradiation condition [10]. 


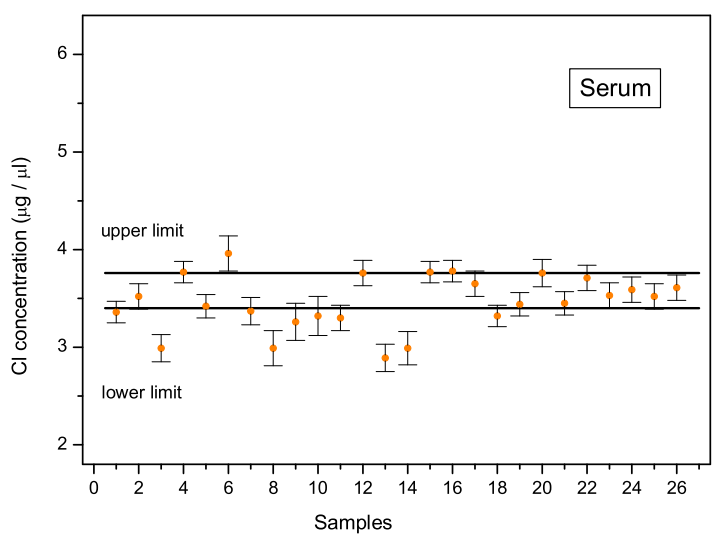

FIG. 3: Chlorine concentration results in serum samples; the horizontal lines represent the reference interval from [2].

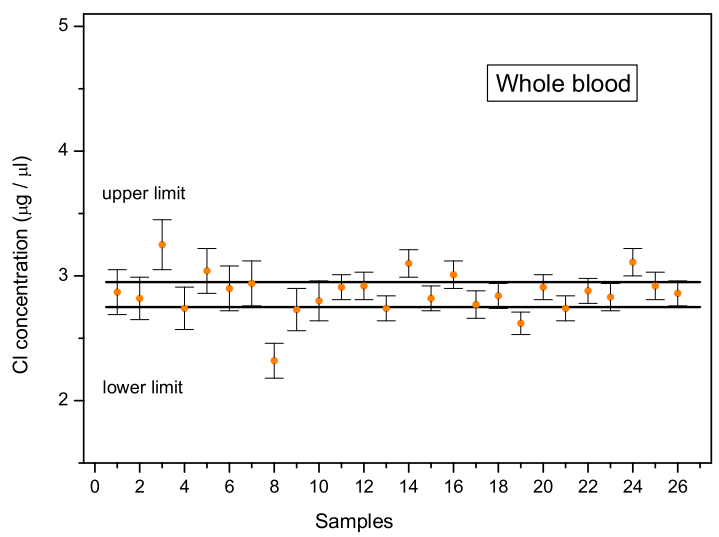

FIG. 4: Chlorine concentration results in whole blood samples; the horizontal lines represent the indicative interval proposed in this work.

\section{DISCUSSION}

In this study the ANAA technique was applied to analyze $\mathrm{Cl}$ in whole blood of a selected group of individuals, for the proposition of an indicative interval for its reference value, to provide information which can help in diagnosis of patients. Although the statistical treatment of reference values a reasonably estimate must be obtained [14], these preliminary results show that the indicative interval obtained for $\mathrm{Cl}$ in serum from the nuclear analysis $\left(3.41-3.69 \mu g \cdot \mu l^{-1}\right)$ is in agreement with the reference value adopted $\left(3.44-3.76 \mu g \cdot \mu l^{-1}\right)$ [2] suggesting that this nuclear methodology can be used to analyze other elements in serum and blood. Of course, more systematic and large scale studies are needed to establish reference values using ANAA aiming its application, in the future, for studying in more details reference values of common defi- ciencies in Brazilian population helping their diagnostics.

Relating to the use of this nuclear methodology to performed hematological examinations some advantages could be appointed: it is not necessary to performed the serum-plasma separation; it permits the simultaneous determination of several elements concentrations; it allows the sample storage for future reexamination, for long periods, without the need of refrigeration and due the short time irradiation and the use of small amounts of biological material low activity is induced, so any specific shielding is necessary after a few days. When ANAA is compared with another nuclear technique, the Instrumental Neutron Activation Analysis (INAA) [15], there are also advantages because the absolute method does not require standards which are imported and expensive besides the comparative method demand much more time, mainly when elements of short half-life are involved due to the necessity to analyze the standards and the sample separately and as some of them can decay before being gamma counted, several irradiation usually must be done. Considering the advantages appointed it is possible to perform clinical analysis in whole blood in an agile, fast and economic way using ANAA.

Other techniques such as PIXE (Particle Induced Xray Emission), ETAAS (Electrothermal Atomic Absorption Spectrometry), Q-ICP-MS (Quadrupole Inductively Coupled Plasma Mass Spectrometry) and TXRF (Total Reflection Xray Fluorescence), for example, have been extensively used for reference value determination, in whole blood, mainly for elements which present the highest potential risk for health, such as, $\mathrm{Cd}, \mathrm{Hg}, \mathrm{Pb}$ (pollutants) as well as $\mathrm{As}, \mathrm{Cr}$ and $\mathrm{Ni}$ (carcinogenic metals) [16], however no reference value for $\mathrm{Ch}$ lorine or other elements in whole blood have been measured for medical diagnosis studies, particularly from inhabitants of the Brazil. Nowadays, measurements of Iron in whole blood are in progress [17], using this methodology, aiming to standardize the nuclear procedure for Iron determination in whole blood, for diagnosis and prediction of Iron deficiency (ane$\mathrm{mia}$ ), that have high prevalence in Brazilian population $(60 \%)$ [18].

\section{CONCLUSIONS}

The $\mathrm{Cl}$ concentration has been measured in serum by using ANAA technique and the result obtained is in agreement with the reference value adopted. This suggests that this nuclear methodology can be applied for the proposition of interval value for all elements activated with neutrons, both in serum and whole blood. The limitation of this method it is the necessity to have access to a nuclear reactor or other types of neutron sources to perform the activation in the samples. However, a small size prototype of neutron irradiator is being developed [19], so we hope that in the future it can be used outside the reactor premises to perform these analyses. 


\section{Acknowledgments}

The authors would like to thank the staff for the technical assistance during the blood collection at Paulista Blood Bank.
This works has been supported by CNPq and FAPESP.
[1] O. Vesterberg, et al. International project reference values for concentrations of trace elements in blood and urine - TRACY. Scand Work Environ Health vol.19 (1993).

[2] J. P. Fréjaville, and P. Kamoun (eds). Guide des examens de laboratoire, Paris, Flammarion (1981).

[3] S. S. Raphael (ed). Lynch's medical laboratory technology, 4.ed., Philadelphia: Saunders (1983).

[4] C. B. Zamboni, I. M. M. A. Medeiros, F. A. Genezini, A. C. Cestari, and J. T. Arruda-Neto, Proceedings of the V ENAN (RJ, Brasil, 2000).

[5] L. C. Oliveira, C. B. Zamboni, A. M. G. Figueiredo, A. C. Cestari, and J. T. Arruda-Neto, Proceedings of the III ENBN (Gramado, Brasil, 2001).

[6] L. C. Oliveira, C. B. Zamboni, A. C. Cestari, L. Dalaqua Jr., M. V. G. Manso, A. M. G. Figueiredo, and J. T. Arruda-Neto, Rev. Bras. Pesq. Des. 4, 1035 (2002).

[7] L. C. Oliveira, C. B. Zamboni, F. A. Genezini, A. M. G. Figueiredo, and G. S. Zahn, Proceedings of the MARC VI (HI, USA, 2003).

[8] L. C. Oliveira, C. B. Zamboni, G. S. Zahn, M. P. Raele, and M. A. Maschio, Brazilian Journal of Physics 34, 811 (2004).

[9] L. C. Oliveira, C. B. Zamboni, L. Dalaqua Jr., and M. Saiki, Proceedings of the 11th MTAA (Guildford, England, 2004).
[10] C. B. Zamboni, L. C. Oliveira, and L. Dalaqua Jr., Proceedings of the ANES (Miami Beach, EUA, 2004).

[11] U. D. Bitelli, M.Sc. Thesis, (Instituto de Pesquisas Energéticas e Nucleares, São Paulo, 1988).

[12] P. Gouffon, Manual do programa IDeFix. (Instituto de Física da Universidade de São Paulo, Laboratório do Acelerador Linear, São Paulo, 1987).

[13] J. A. G. MEDEIROS, Private communication, (Instituto de Pesquisas Energéticas e Nucleares - IPEN, São Paulo, 2002).

[14] A. H. Reed, R. Henry, and W. B. Mason, Clinical Chemistry 17, 275 (1971).

[15] M. D. Glascok, In: Electronic Annals: http://www.missouri.edu/ glascok/naa_over.htm. (Accessed on Mar 2004).

[16] International Federation of Clinical Chemistry, Clinica Chimica Acta, 137, 97F (1984).

[17] P. S. Lins, M.Sc. Thesis (Universidade de Santo Amaro, São Paulo, 2004).

[18] S. S. Queiroz, et al., Jornal de Pediatria 76, s298 (2000).

[19] K. Shtejer-Diaz, C. B. Zamboni, G. S. Zahn, and J. Y. ZevallosChávez. Appl. Radiat. Isot. 59, 263 (2003). 